\title{
Characterization of a logarithmic spike timing encoding scheme for a $4 \times 4$ tin oxide gas sensor array
}

\author{
Kwan Ting $\mathrm{Ng}^{1,2}$, Bin $\mathrm{Guo}^{2}$, Amine Bermak ${ }^{2}$, Dominique Martinez ${ }^{3}$, and Farid Boussaid ${ }^{1}$ \\ ${ }^{1}$ School of Electrical, Electronic and Computer Engineering \\ The University of Western Australia, Perth, WA, Australia \\ ${ }^{2}$ Department of Electronic and Computer Engineering \\ Hong Kong University of Science and Technology, Clear Water Bay, Kowloon, Hong Kong \\ ${ }^{3}$ Cortex Project, LORIA, Campus Scientifique, B.P. 239, 54506 Vandoeuvre-l'es-Nancy, France \\ Email: kashng@ee.uwa.edu.au
}

\begin{abstract}
In this paper, the performance of a logarithmic spike timing encoding scheme for gas sensor is analyzed. Utilizing the non-linear (power law) relationship between tin oxide $\left(\mathrm{SnO}_{2}\right)$ gas sensor sensitivity and gas concentration, we designed and fabricated a time domain readout circuit for a $4 \times 4 \mathrm{SnO}_{2}$ gas sensor array. A unique pattern is generated by the readout scheme for each gas, irrespective of the gas concentration. This pattern is used as the signature of the gas. Gas identification is achieved by matching the generated signature with pre-stored reference signatures. The design was implemented in standard CMOS technology. Reported experimental results demonstrate the fabricated gas sensor exhibits the detection accuracy comparable to computationally expensive classifiers.
\end{abstract}

\section{INTRODUCTION}

Many researchers have investigated Electronic Nose (EN) systems with an array of gas sensors, but currently only complex and expensive solutions are available in the market. These EN systems utilize commercial gas sensors for sensing and computer platforms for processing. Conventionally, classifiers including the $\mathrm{K}$ nearest neighbors (KNNs), multilayer perceptron (MLP), radial basis function (RBF), Gaussian mixture model (GMM) and probabilistic principal component analysis (PPCA) are used to separate the uncorrelated data from the sensor in order to achieve gas identification. However, applications in distributed sensor networks, real-time in-field measurements, hand-held devices and aerospace industries require small-size, low-power and 'smart' (possessing basic or advanced computation ability) EN systems that does not require computers for data processing. Besides, a major drawback of discrete gas sensors is either lack of selectivity or fixed gas detection. An integrated gas sensor array, on the contrary, can increase the selectivity and detect multiple target gases with a single device [1] - [6].

We have previously reported a bio-inspired recognition algorithm based on logarithmic spike timing with rank order encoding for a $4 \times 4 \mathrm{SnO}_{2}$ gas sensor array [7]. The proposed algorithm has significant advantage that enables nose-on-chip solution. In this paper, we report the experimental results characterizing the logarithmic spike timing circuit and evaluates its performance. We have tested it with the fabricated $\mathrm{SnO}_{2}$ gas sensor array. Efficient gas discrimination results have been demonstrated. The development of the encoding/identification system and the experimentally tested results will be presented in the following sections. Section II briefly introduces the logarithmic spike timing encoding scheme. Section III describes the VLSI implementation. Section IV presents the experiment results and characterizations of our scheme where section $\mathrm{V}$ concludes the paper.

\section{LOGARITHMIC SPIKE TIMING ENCODING SCHEME}

The steady state change in the conductance (sensitivity) $x$ of a metal oxide gas sensor is defined by the ratio between the steady state resistance $R_{s}$ and the baseline resistance $R_{o}$ and it can be approximated by the power law relationship with respect to gas concentration.

$$
x \approx \frac{R_{s}}{R_{o}}=\alpha C^{\gamma}
$$

where $\alpha$ and $\gamma$ are coefficients that depend upon the gas and the sensing material. Considering a neural network with spiking neurons such that, when gas $j$ is sampled at time $t=0$, the input neuron $i$ (connected to sensor $i$ ) will fire its first spike at time $t_{i j}$ given by:

$$
t_{i j}=\frac{\log x_{i j}}{\gamma_{i j}}
$$

where $x_{i j}$ is the steady state change in conductance of sensor $i$ and $\gamma_{i j}$ is the parameter fitted for odor $j$ respectively. Imaging the $4 \times 4$ integrated gas sensor array act as 16 input neurons. The output spikes of the 16 neurons will constitute a spike train with each spike time delay as $t_{i j}$. The interval $\Delta_{p q}$ between two individual spikes, i.e. the spikes generated by the $p^{t h}$ and 
the $q^{\text {th }}$ neurons, in this train can be calculated as:

$$
\begin{aligned}
\Delta_{p q} & =t_{p}-t_{q} \\
& =\frac{\log x_{p}}{\gamma_{p}}-\frac{\log x_{q}}{\gamma_{q}} \\
& =\frac{\log \alpha_{p}+\gamma_{p} \log C}{\gamma_{p}}-\frac{\log \alpha_{q}+\gamma_{q} \log C}{\gamma_{q}} \\
& =\frac{\log \alpha_{p}}{\gamma_{p}}-\frac{\log \alpha_{q}}{\gamma_{q}}
\end{aligned}
$$

The above equation indicates that $\Delta_{p q}$ is independent of the gas concentration $C$. In other words, the relative position of spikes in the generated spike train is unchanged for each individual gas, regardless the concentration variation. So one can obtain a unique spike train pattern for each analyte gas. Simple rank order recognition can thus be used for gas discrimination, which can reduce the computational complexity compared to regular pattern classification methods. In summary, one can use a logarithmic transformation to convert the output of the gas sensor array into spike trains and then use rank order recognition to perform gas classification.

\section{VLSI IMPLEMENTATION}

The proposed identification scheme has been realized by VLSI implementation. Fig. 1 shows major building blocks of the resistance to logarithmic timing spike conversion to implement the proposed algorithm. The sensor is modeled by

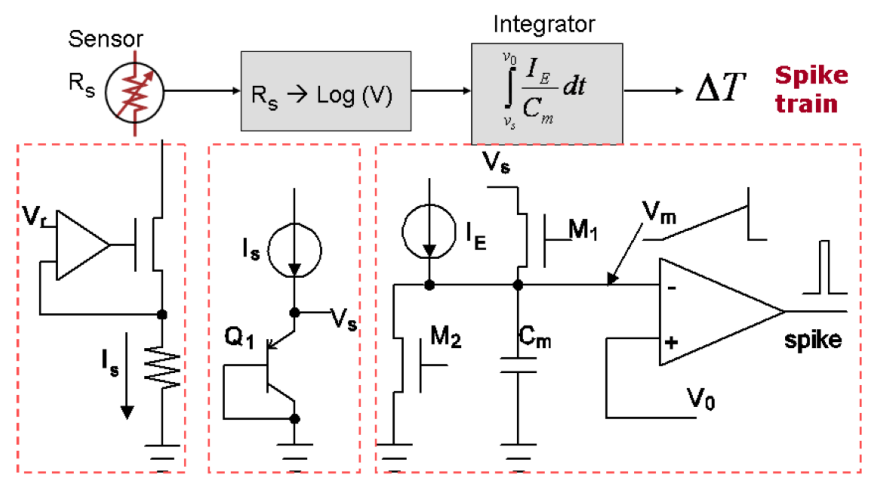

Fig. 1. Major building blocks of resistance to logarithmic timing spike conversion

a resistor in the figure. It is converted to current given by:

$$
I_{s}=\frac{V_{r}}{R_{s}}
$$

This current is copied and fed into a PNP bipolar junction transistor in order to generate a logarithmic output voltage $V_{s}$ :

$$
\begin{aligned}
V_{s} & =V_{T} \ln \frac{I_{s}}{I_{E S}} \\
& =V_{T} \ln \frac{V_{r}}{I_{E S} \cdot R_{s}}
\end{aligned}
$$

where $V_{T}$ and $I_{E S}$ are the thermal voltage and reverse saturation current. A similar circuit is designed to convert the baseline resistance $R_{o}$ to voltage $V_{o}$. In addition to this circuit, a voltage shifter is used for the $V_{o}$ generation to ensure that $V_{o}>V_{s}$

A spike is then generated at the time $t$ which is proportional to the voltage difference between $V_{s}$ and $V_{o}$.

$$
I_{E} \cdot t=V_{T} \ln \frac{V_{r}}{I_{E S}}+V_{T} \ln \frac{R_{s}}{R_{0}}
$$

where $t$ is the timing delay for a neuron to generate a spike after the integration starts, or the time-to-the-first-spike (TFS).

By setting $I_{E}$ according to the value of $\gamma$ from (1), (6) can be rewritten as:

$$
\begin{aligned}
\gamma \cdot t & =X+Y \ln \frac{R_{s}}{R_{0}} \\
t & =\frac{X}{\gamma}+\frac{Y}{\gamma} \ln \frac{R_{s}}{R_{0}}
\end{aligned}
$$

where $X$ and $Y$ are constants determined by the circuit parameters and are the same for all neurons. By characterizing the fabricated gas sensor, we have discovered that the dynamic range of $\gamma$ is up to $62 \mathrm{~dB}$ among the 3 target gases including hydrogen $\left(\mathrm{H}_{2}\right)$, ethanol and carbon monoxide $(\mathrm{CO})$. However, for each individual gas, the dynamic range of $\gamma$ is only about $16 \mathrm{~dB}$. To reduce the complexity of our scheme, we normalize the $\gamma$ by taking the ratio between $\gamma$ and the minimum $\gamma$ across the entire array of the gas. Setting $I_{E}$ to be $a=\gamma / \min .(\gamma)$, the TFS of the sensor becomes:

$$
t=\frac{B}{a}+\frac{A}{a} \ln \frac{R_{s}}{R_{0}}
$$

where A and B are constants depends on the circuit parameters and $\min .(\gamma)$. The resolution of the current source depends on the sensor characterization and it will be discussed in the next section. It can be shown from (8) that the spike time interval $\Delta_{p q}$ between neurons $p$ and $q$ can be expressed by:

$$
\begin{aligned}
\Delta_{p q} & =t_{p}-t_{q} \\
& =\frac{B}{a_{p}}+\frac{A}{a_{p}} \ln \frac{\left(R_{s}\right)_{p}}{\left(R_{0}\right)_{p}}-\left(\frac{B}{a_{q}}+\frac{A}{a_{q}} \ln \frac{\left(R_{s}\right)_{q}}{\left(R_{0}\right)_{q}}\right) \\
& =\left(\frac{B}{a_{p}}-\frac{B}{a_{q}}\right)+\left(\frac{A \cdot \ln \alpha_{p}}{a_{p}}-\frac{A \cdot \ln \alpha_{q}}{a_{q}}\right)
\end{aligned}
$$

The above calculation shows that the gas concentration has no effect to the spike train generated by the proposed circuit.

\section{EXPERIMENTS AND RESULTS}

An integrated $4 \times 4$ tin oxide gas sensor array was designed and fabricated using our in-house $5 \mu \mathrm{m}$ 1-metal, 1-poly CMOS process. Table I summarizes the gas sensor array characterization results including the values of baseline resistances and $a_{i j}$ for sensor $i$ exposing to gas $j$.

In VLSI implementation, the current source $I_{E}$ for neuron $i$ is set to be $a_{i j}$ and it is the only parameter that can be varied in the circuit. The precision of $I_{E}$ directly affects the performance of the proposed approach. As rank order is used in this encoding scheme, our approach is relatively robust because false alarm is only occurred when spikes drift causing them to be swapped. The drift of spikes is mainly due to 
TABLE I

GAS SENSOR ARRAY CHARACTERIZATION

\begin{tabular}{c|l|cccc}
\hline \hline & & Col1 & Col2 & Col3 & Col4 \\
\hline \multirow{3}{*}{$R_{o}(k \Omega)$} & Row1 & 22.3 & 240 & 26.95 & 17.08 \\
& Row2 & 24.94 & 117.88 & 16.81 & 13.37 \\
& Row3 & 22.61 & 233.9 & 16.59 & 18.14 \\
& Row4 & 23.47 & 155.02 & 29.37 & 19.85 \\
\hline \multirow{3}{*}{$a_{i 1}$ (Hydrogen) } & Row1 & 1.001 & 2.506 & 2.338 & 1.658 \\
& Row2 & 1.000 & 1.641 & 2.220 & 1.600 \\
& Row3 & 1.193 & 2.506 & 2.475 & 1.847 \\
& Row4 & 1.225 & 1.961 & 2.149 & 1.886 \\
\hline \multirow{3}{*}{$a_{i 1}$ (Ethanol) } & Row1 & 1.558 & 6.674 & 1.139 & 1.000 \\
& Row2 & 1.302 & 6.233 & 1.000 & 1.093 \\
& Row3 & 2.535 & 6.674 & 1.116 & 1.209 \\
& Row4 & 2.651 & 6.488 & 1.116 & 1.279 \\
\hline \multirow{3}{*}{$a_{i 1}(\mathrm{CO})$} & Row1 & 1.000 & 2.778 & 2.111 & 1.667 \\
& Row2 & 1.444 & 2.055 & 1.389 & 1.555 \\
& Row3 & 1.278 & 2.778 & 1.389 & 1.778 \\
& Row4 & 1.722 & 2.389 & 2.222 & 1.889 \\
\hline \hline
\end{tabular}

the quantization error of $I_{E}$ in the proposed readout circuit. Therefore, the resolution of $I_{E}$ has to be designed carefully. A high resolution $I_{E}$ would enhance the robustness of the scheme. However, this trades off with the chip area since 16 current sources are required for the entire readout circuit. The drift of spike $i$ can be expressed by:

$$
\begin{aligned}
\delta t_{i} & =\left|t_{i}^{\prime}-t_{i}\right| \\
& =\frac{B+A \ln \frac{R_{s i}}{R_{0 i}}}{I_{E i}+\delta I_{E i}}-\frac{B+A \ln \frac{R_{s i}}{R_{0 i}}}{I_{E i}}
\end{aligned}
$$

where $\delta I_{E i}$ is the error caused by the quantization error. Error free identification can be ensured if the maximum drift of all spikes,$\delta t_{\max }$ is less than half of the minimum time spike interval $\Delta_{\min }$ of the spike train as illustrated in fig. 2 .

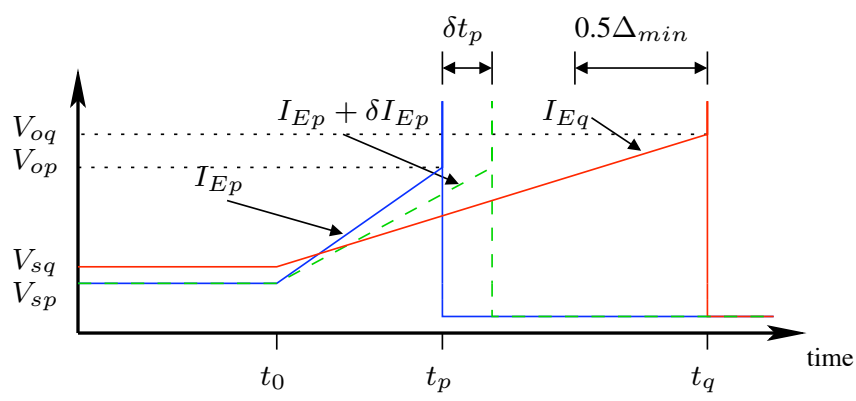

Fig. 2. Drift

Assume $t_{p}$ and $t_{q}$ are the two closest TFS among the entire spike train, $\Delta_{\min }$ is defined by

$$
\Delta_{\min }=\left|t_{p}-t_{q}\right|
$$

Hence, $\delta t_{\max }$ is given by:

$$
\begin{aligned}
\delta t_{\max } & <0.5 \Delta_{\min } \\
& <\left|\frac{B}{I_{E p}}+\frac{A}{I_{E p}} \ln \frac{R_{s p}}{R_{0 p}}-\left(\frac{B}{I_{E q}}+\frac{A}{I_{E q}} \ln \frac{R_{s q}}{R_{0 q}}\right)\right|
\end{aligned}
$$

By solving (10) and (12), we are able to compute $\delta I_{E i}$. As a result, we found that an 8 -bit current source is enough to provide a robust readout circuit. In the VLSI implementation, however, a design margin is chosen for $I_{E i}$ with 10-bit to further enhance the robustness because the current source is the most critical component in the scheme.

The proposed readout scheme has been designed and fabricated by Chartered $0.35 \mu \mathrm{m}$ 2-poly 4-metal standard CMOS process with its microphotograph shown in Fig.3. The whole chip occupies an area of $2.72 \times 1.888 \mathrm{~mm}^{2}$ including the bonding pads.

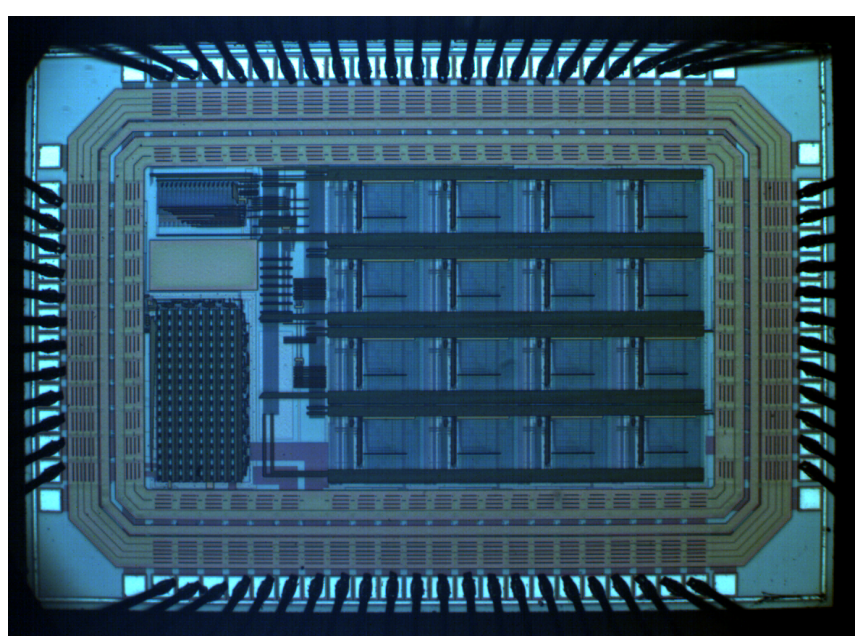

Fig. 3. Microphotograph of the proposed readout circuit

The circuit was tested with data obtained from the sensor array. It was exposed to gases $\left(\mathrm{H}_{2}\right.$, ethanol and $\left.\mathrm{CO}\right)$ at different concentrations. Fig. 4 shows the spiking sequence of $\mathrm{CO}$ at increasing concentration. This shows our proposed scheme is insensitive to concentration variation.

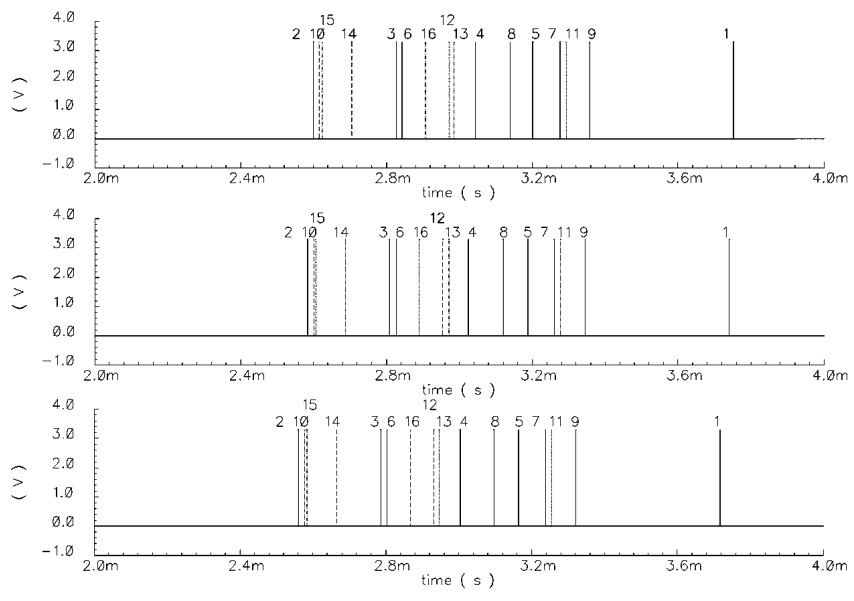

Fig. 4. Spike train of $\mathrm{CO}$ at 50, 100, 150ppm from top to bottom

Fig. 5 depicts the spike rank orders corresponding to the sensor number in the array for the 3 analyte gases. Each tested gas generates a unique rank order as described in the previous subsections. The radius in the figure represents the sensor number, while the number on the circumference represents the 
rank of this spike in the spike train. For the 3 tested gases, 3 different rank order patterns can be obtained from the proposed neuron array, which can be used for recognition of such gas.

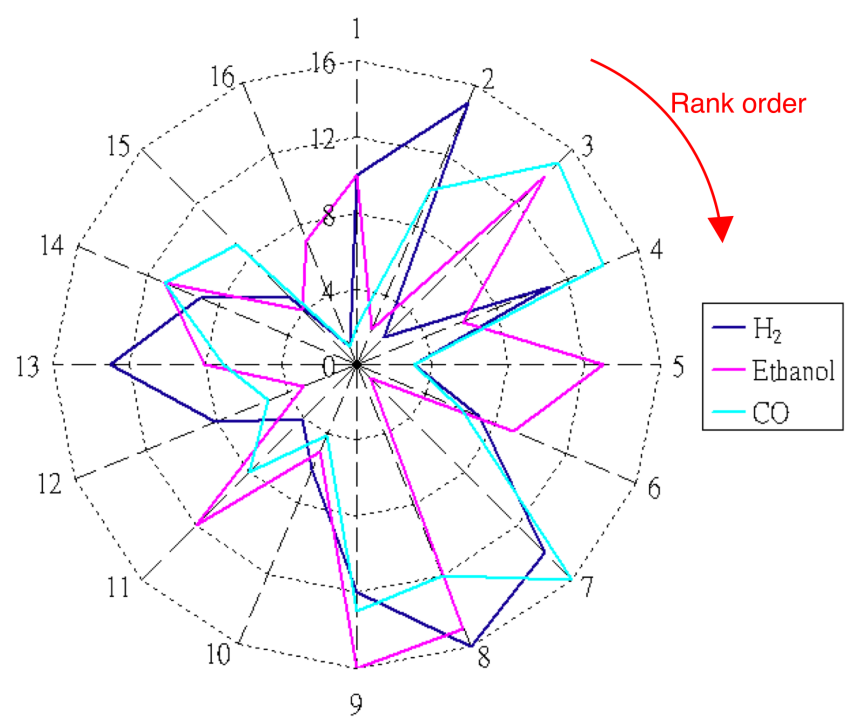

Fig. 5. The rank order for different gases

Table II summarizes the correct detection rate of the proposed approach and other classifiers. This rate generally increases with the number of principal components (PCs). However, the complexity of the algorithm also increases. In our work, we have achieved a successful gas detection rate that is comparable to classifiers with 4 PCs.

TABLE II

COMPARISION OF ACCURACY (\%) BETWEEN THIS WORK AND OTHER CLASSIFIERS WITH DIFFERENT PRINCIPLE COMPONENTS

\begin{tabular}{|c|c|c|c|c|c|c|c|}
\hline No. of PCs & 2 & 3 & 4 & 5 & 6 & 7 & 8 \\
\hline KNN & 79.1 & 86.4 & 88.6 & 87.7 & 88.2 & 89.1 & 89.1 \\
\hline MLP & 81.4 & 88.2 & 91.8 & 90.5 & 93.2 & 93.6 & 92.3 \\
\hline RBF & 70.9 & 75.5 & 86.4 & 86.8 & 81.8 & 83.2 & 82.3 \\
\hline GMM & 75.0 & 86.4 & 90.9 & 94.5 & 90.0 & 91.8 & 90.9 \\
\hline PPCA & 70.5 & 81.8 & 84.5 & 84.1 & 79.1 & 79.1 & 79.1 \\
\hline This work & \multicolumn{7}{|c|}{88.6} \\
\hline
\end{tabular}

\section{CONCLUSION}

A logarithmic spike timing encoding scheme is presented in this paper. It has been successfully tested with data from a $4 \times 4$ tin oxide gas sensor array. This encoding scheme can generate unique spike sequence for different gases. The unique spike sequence is independent on the gas concentration, thus can be used to discriminate between different gases with concentration invariant result. Using a relatively simple structure, this encoding scheme illustrates powerful computation capability which is comparable to computer based classifiers.

\section{REFERENCES}

[1] K. Persaud and G. H. Dodd, "Analysis of discrimination mechanisms of the mammalian olfactory system using a model nose", Nature, Vol. 299, 1982, pp. 352-355.
[2] G. Hagleitner, A. Hierlemann, D. Lange, A. Kummer, N. Kerness, O Brand and H. Baltes, "Smart single-chip gas sensor microsystem". Nature, Vol. 414, Nov 2001, pp.293 - 296.

[3] D. Martinez, "On the right scent", Nature, 445, 2007, pp. 371-372, Jan. 2007 (News and Views).

[4] M. Y. Afridi, J. S. Suehle, M. E. Zaghloul, D. W. Brning, A. R. Hefner, R. E. Cavicchi et al., "A Monolithic CMOS Microhotplate-Based Gas Sensor System”, IEEE Sensors Journal, 2 (6), 2002, pp. 644-655.

[5] C. Cane, I. Gracia, A. Gotz, L. Fonseca, E. Lora-Tamayo, M. C. Horrillo, I. Sayago, J. I. Robla, J. Rodrigo and J. Gutierrez, "Detection of Gases with Arrays of Micromachined Tin Oxide Gas Sensors", Sensors and Actuators B, 65, 2000, pp. 244-246.

[6] U. Frey, M. Graf, S. Taschini, K.-U. Kirstein, C. Hagleitner, A. Hierlemann, and H. Baltes, "A Digital CMOS Micro-Hotplate Array for Analysis of Environmentally Relevant Gases”, ESSCIRC 2004, Sep 2004, pp. 299-302.

[7] Guo, B., Bermak, A., Ambard, M., Martinez, D., "A $4 \times 4$ Logarithmic Spike Timing Encoding Scheme for Olfactory Sensor Applications, " ISCAS 2007, pp.3554-3557, 27-30 May 2007 